\title{
The mechanism of energy loss and transition in a flow with submerged vegetation
}

\author{
W.X. Huai ${ }^{\text {a,* }}$, J. Han ${ }^{\text {a }}$, C. Geng ${ }^{\text {a }}$, J.F. Zhou ${ }^{\text {b }}$, Y.H. Zeng ${ }^{\text {a }}$ \\ a State Key Laboratory of Water Resources and Hydropower Engineering Science, Wuhan University, Wuhan 430072, China \\ b Institute of Mechanics, Chinese Academy of Science, Beijing 100192, China
}

\section{A R T I C L E I N F O}

\section{Article history:}

Received 29 August 2009

Received in revised form 16 March 2010

Accepted 16 March 2010

Available online 25 March 2010

\section{Keywords:}

Energy balance

Energy loss

Energy transition

Steady uniform flow

Submerged vegetation

\begin{abstract}
A B S T R A C T
The mechanism of energy balance in an open-channel flow with submerged vegetation was investigated. The energy borrowed from the local flow, energy spending caused by vegetation drag and flow resistance, and energy transition along the water depth were calculated on the basis of the computational results of velocity and Reynolds stress. Further analysis showed that the energy spending in a cross-section was a maximum around the top of the vegetation, and its value decreased progressively until reaching zero at the flume bed or water surface. The energy borrowed from the local flow in the vegetated region could not provide for spending; therefore, surplus borrowed energy in the non-vegetated region was transmitted to the vegetated region. In addition, the total energy transition in the cross-section was zero; therefore, the total energy borrowed from the flow balanced the energy loss in the whole cross-section. At the same time, we found that there were three effects of vegetation on the flow: turbulence restriction due to vegetation, turbulence source due to vegetation and energy transference due to vegetation, where the second effect was the strongest one.
\end{abstract}

Crown Copyright @ 2010 Published by Elsevier Ltd. All rights reserved.

\section{Introduction}

Vegetation in a channel may result in a higher water level during flooding. In addition, it is beneficial to the river ecosystem because it provides a proper habitat for aquatic animals. Therefore, the turbulent structure of vegetated flow has always been of interest to hydraulicians. Some researchers have regarded aquatic vegetation as parts of roughness in the riverbed and studied the vegetative resistance acting on the flow $[4,6,9]$, but they usually did not describe how the vegetation influenced the flow in detail. In recent years, more researchers have been interested in the vertical distributions of the stream-wise velocity [7,10] and Reynolds stress [2,5,14]. Ghisalberti and Nepf [3] further studied the coherent structure of flow resulting from submerged vegetation and pointed out [8] that the vertical exchanges of mass, momentum and energy were dominated by two scales of vortices: the shear-scale vortices generated and stem-scale vortices (Fig. 1). However, the mechanism of energy exchange in a flow with vegetation has not been studied yet. We attempt to investigate this mechanism in the present paper.

Bakhmeteff and Allan [1] studied the vertical distribution of energy and presented the process of energy loss and transition in an openchannel flow. They gave the expressions of energy borrowing, energy spending and energy transition, and built a balance relationship

\footnotetext{
* Corresponding author. Tel.: +86 27 68772211; fax: +86 2768772310 E-mail address: wxhuai@whu.edu.cn (W.X. Huai).
}

between the expressions. They pointed out that (1) at any point in the flow, the energy supplied by local flow is always equal to the sum of local energy loss and transition, (2) the energy dissipates mostly in the region near the flume bed, and thus this region needs excess energy transferred from other regions, and (3) in the whole cross-section, the total energy transition is zero and thus the total energy borrowed from the flow is equal to the total energy spending. On the basis of their research, the present paper analyzes how the energy supplied by the flow distributes, transports and dissipates in the flow with submerged vegetation, and tries to build a balance relationship among the energy borrowing, energy spending and energy transition. Three effects of vegetation acting on the flow are then discussed-the restriction on flow, turbulence due to vegetation, and energy transference.

\section{Background}

A two-dimensional steady uniform flow with submerged vegetation in a straight open channel is considered in this paper. In a control volume (CV) of $V=\mathrm{d} x \times 1 \times \mathrm{d} z$ in the flow, the spatially averaged $x$ equation (Fig. 1 ) is $[7,13]$

$\frac{d \tau(z)}{d z}=-\gamma S+f_{\mathrm{cd}}(z)$

where $z$ is the vertical coordinate and $\tau(z)=\tau_{x z}(z)+\tau^{\prime}(z)$ is the shear stress, which consists of Reynolds stress $\tau_{x z}(z)$ and viscosity stress $\tau^{\prime}(z)$. Although the latter is usually neglected [8,11], this paper 


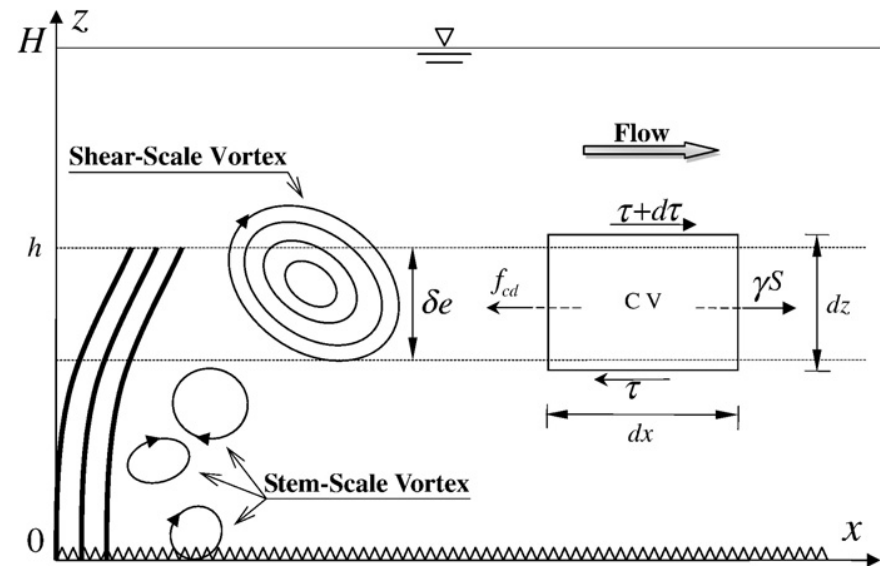

Fig. 1. The control volume in the flow with submerged vegetation. The conceptions of the shear-scale and stem-scale vortices come from [8]. Notice that in this figure, the shear stresses $\tau+\mathrm{d} \tau$ and $\tau$ are surface forces, and both $\gamma S$ and $f_{\mathrm{cd}}$ are body forces per unit volume [10]. $\mathrm{d} \tau / \mathrm{d} y$ in the control equation is also a body force per unit volume and is given by $((\tau+\mathrm{d} \tau)-\tau) \times \mathrm{d} x \times 1 /(\mathrm{d} x \mathrm{~d} z \times 1)$.

considers it when calculating the shear stress. $\gamma$ is the specific weight of water and $S$ is the energy slope, which is equal to the bed slope in the case of uniform flow. $f_{\mathrm{cd}}(z)$ is the general drag force due to vegetation, and is defined as

$f_{\mathrm{cd}}(z)= \begin{cases}0 & h<z \leq H \\ \frac{1}{2} \rho C_{\mathrm{d}} a u^{2}(z) & 0 \leq z \leq h\end{cases}$

where $C_{\mathrm{d}}$ is the drag coefficient, $u$ is the stream-wise velocity, $H$ is the water depth, and $h$ is the plant height. This study will not strictly distinguish flexible and rigid vegetation and generally uses $h$ to represent the height of rigid vegetation or the average height of flexible vegetation. $a$ is the vegetation density, which is defined by $a=b /(L x L y)$, where $b$ is the front width of a single plant and $L x$ and $L y$ are the spaces between plants along longitudinal and transverse directions [8,12].

Shimizu and Tsujimoto [11] used rigid cylinders with equal height and diameter in a square pattern to simulate vegetation, and gave results for the velocity and Reynolds stress of four runs. The present paper uses the computational results of Run A71 in [11] to discuss the energy balance in the open-channel flow with submerged vegetation. The parameters of Run A71 were $H=8.95 \mathrm{~cm}, h=4.6 \mathrm{~cm}, S=8.86 \%$, $b=0.15 \mathrm{~cm}$, a length of the square space between plants of $2.0 \mathrm{~cm}$, $a=0.0375 \mathrm{~cm}^{-1}$, a depth-averaged velocity $U=33.05 \mathrm{~cm} / \mathrm{s}$, and a drag coefficient $C_{\mathrm{d}}=1.0-1.5$. As [11] neglected the viscosity stress and did not give the value of water viscosity, the present paper arbitrarily assumes the kinematic viscosity of flow is $1.0 \times 10^{-2} \mathrm{~cm}^{2} / \mathrm{s}$ (i.e., the temperature is $20^{\circ} \mathrm{C}$ ).

\section{Mechanism of energy loss and transition}

\subsection{Energy borrowed from local flow}

We define $h_{f}=E_{1}-E_{2}$ as the head loss between two random sections 1 and 2 in a uniform flow and $\Delta l$ as the distance between the two sections, where $E_{1}$ is the total head at section 1 while $E_{2}$ is the one at section 2. Therefore, the energy slope of the flow $S=h_{f} / \Delta l=\left(E_{1}-E_{2}\right) / \Delta l$ represents the energy borrowing per unit weight liquid in the unit flow path, and $\gamma S$ is the energy borrowing per unit volume liquid in the unit flow path. The energy borrowing per unit volume of local flow during a unit time interval is defined as the product of $\gamma S$ and local velocity $u$ :

$W_{\mathrm{b}}=\gamma S u$.
From the controls of Eqs. (1) and (3), the energy borrowed from the local flow is

$W_{\mathrm{b}}=-u \frac{d \tau}{d z}+u f_{\mathrm{cd}}$

\subsection{Energy spending}

The energy spending in the vegetated flow can be divided into the spending to overcome flow resistance and the spending to overcome the vegetation drag. Bakhmeteff and Allan [1] gave the former as

$W_{\mathrm{sW}}=\tau \frac{d u}{d z}$

The drag force of vegetation in the volume is defined as

$F_{\mathrm{v}}=\frac{1}{2} \rho C_{\mathrm{d}} A u^{2}$

where $A$ is the front area of vegetation in the volume and equal to the product of the plant number in the volume $n$, front width of a single plant $b$ and height of volume $\mathrm{d} z$. During a time period $\mathrm{d} t$, the flow passes through the vegetation with a route distance of $u \mathrm{~d} t$, so the work done by the drag force in a unit volume during a unit time interval is defined as

$W_{\mathrm{sv}}=\left\{\begin{array}{ll}0 & h<z \leq H \\ \frac{\rho C_{\mathrm{d}} A u^{2}}{2 d x d z \times 1 \times d t} \times u d t=\frac{1}{2} \rho C_{\mathrm{d}} a u^{3} & 0 \leq z \leq h\end{array}\right.$,

where $A / V=a$ is the vegetation density [7]. From Eqs. (5) and (7), the energy spending is

$W_{\mathrm{s}}=W_{\mathrm{sw}}+W_{\mathrm{sv}}=\tau \frac{d u}{d z}+u f_{\mathrm{cd}}$

\subsection{Energy transition}

According to [1], $(\tau \mathrm{d} x \times 1) \times u$ is the work done on the lower surface of a CV during a unit time interval or the energy transferred downward from the $\mathrm{CV}$, and $(\tau+\mathrm{d} \tau) \times \mathrm{d} x \times 1 \times(u+\mathrm{d} u)$ is that on the upper surface or the energy transferred downward to the $\mathrm{CV}$; therefore, the total energy transition downward from the $\mathrm{CV}$ is

$$
\begin{aligned}
(\tau d x & \times 1) u-(\tau+d \tau) d x \times 1 \times(u+d u)=-d x \times 1 \\
& \times(\tau d u+u d \tau)-d \tau d u d x \times 1 .
\end{aligned}
$$

Neglecting quantities of high order of smallness $\mathrm{d} \tau \mathrm{d} u \mathrm{~d} x$ and dividing Eq. (9) by the volume $\mathrm{d} x \times 1 \times \mathrm{d} z$ gives

$W_{\mathrm{t}}=-\frac{d(\tau u)}{d z}$

Eq. (10) is the energy transition in a unit volume during a unit time interval and has the same form in flow with or without vegetation.

\subsection{Discussion}

Using the computational results of A71 [11], the energy supplied by the local flow, the energy loss and energy transition are plotted in Fig. 2. All the results are scaled with the averaged cross-sectional energy borrowing $W_{0}=\gamma S U$, where $U$ is the depth-averaged velocity. Fig. 2 indicates that the rule of energy spending and transition in the nonvegetated region is similar to that for an ordinary open-channel flow. The energy borrowed from local flow is always greater than the local energy spending; therefore, surplus energy borrowing will accumulate 


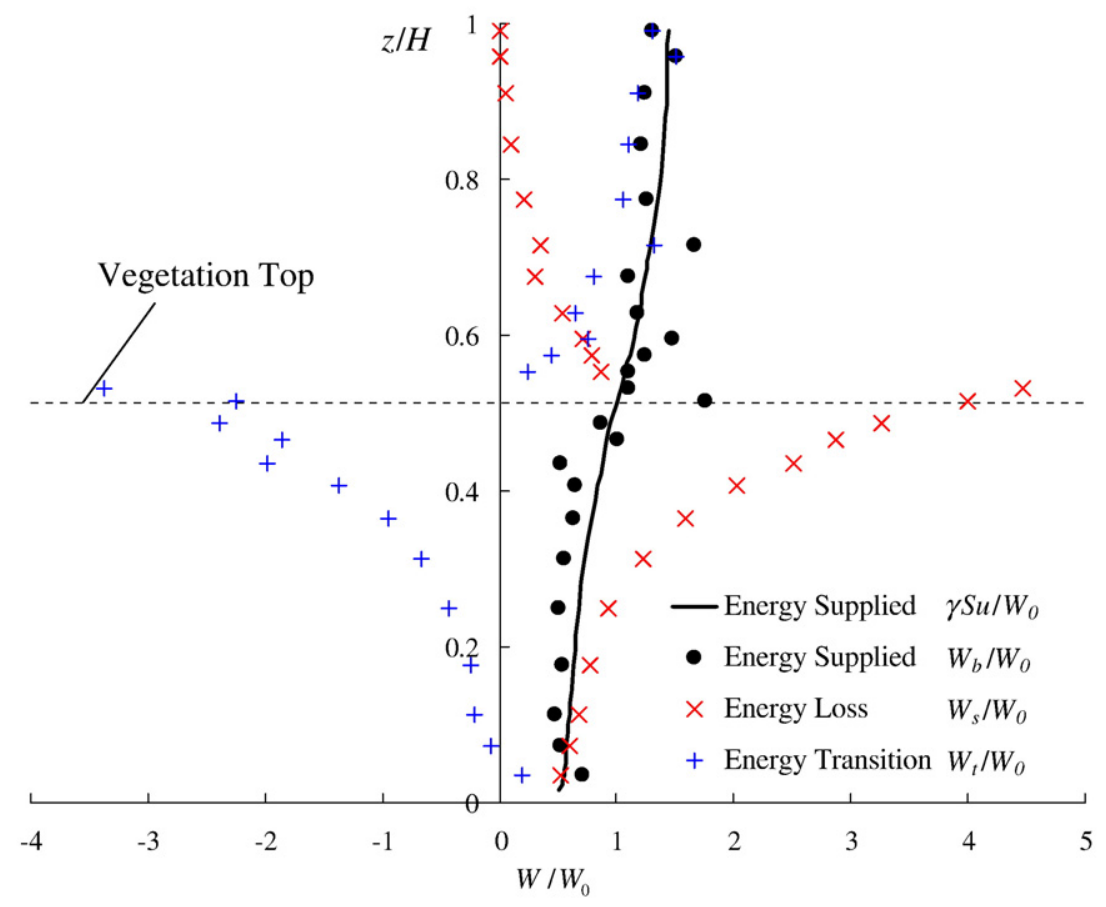

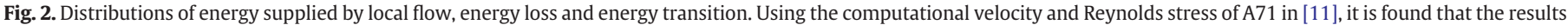
obtained using Eqs. (3) and (4) do not strictly equal each other. The line represents the results of Eq. (3), and the round dots represent those of Eq. (4).

in the non-vegetated region and then be transferred to the vegetated region.

The energy distribution in the vegetated region behaves differently from that in the non-vegetated region:

(1) Because the vegetation results in an additional energy spending, the energy spending is discontinuous and jumps to a maximum at the top of vegetation. Furthermore, the energy spending decreases downward with decreasing velocity. The absolute values of energy spending and transition are greater in the upper vegetated region, which indicates that the vertical exchange of mass and momentum in the region is more intense. This conclusion was also stated by [8].

(2) The value of energy transition is negative and the energy spending is always greater than the energy borrowing. Therefore, surplus energy is transited from the non-vegetated region to cover the requirement for spending in the vegetated region.

(3) In the near-bed region, the viscosity stress is the main shear stress and leads to an increase in energy spending; however, the value is much smaller than the energy loss at the top of the vegetation.

\section{Energy balance}

Using the results of Section 3, this section analyzes the energy balance at a point and in a cross-section, and then discusses the effects of vegetation on flow.

\subsection{Energy balance at a point}

Considering Eqs. (4) and (8), Eq. (10) is rewritten as

$$
\begin{aligned}
W_{\mathrm{t}} & =-u \frac{d \tau}{d z}-\tau \frac{d u}{d z} \\
& =\left(-u \frac{d \tau}{d z}+u f_{\mathrm{cd}}\right)-\left(\tau \frac{d u}{d z}+u f_{\mathrm{cd}}\right) \\
& =W_{\mathrm{b}}-W_{\mathrm{s}}
\end{aligned}
$$

which is:

$W_{\mathrm{b}}=W_{\mathrm{s}}+W_{\mathrm{t}}$

The energy balance in Eq. (12) has the same form as that for flow without vegetation, and the conclusion about the energy balance is similar: the energy supplied by local flow is equal to the sum of local energy loss and local energy transition at a random point in the case of flow with vegetation (Fig. 2). Calculating the plane areas bounded by energy curves and the $z$-axis, we find two interesting results: (1) the total area bounded by the energy transition curve and the $z$-axis is zero and (2) the total area bounded by the energy borrowing curve and the $z$-axis equals that of the energy spending curve. The results imply that in a whole cross-section, the energy borrowing balances with energy spending. The next section illustrates the results in detail.

\subsection{Energy balance in a cross-section}

From Eq. (12), we can deduce the energy balance in a whole crosssection. If we integrate the control Eq. (1) from $H$ downward to $z$, then

$$
\begin{aligned}
\tau & =\gamma(H-z) S+\int_{H}^{z} f_{\mathrm{cd}} d z \\
& =\gamma(H-z) S-F_{\mathrm{cd}}
\end{aligned}
$$

where $F_{\mathrm{cd}}=\int_{z}^{h} f_{\mathrm{cd}} \mathrm{d} z=\int_{z}^{h} f_{\mathrm{cd}} \mathrm{d} z+\int_{h}^{H} f_{\mathrm{cd}} \mathrm{d} z=\int_{z}^{H} f_{\mathrm{cd}} \mathrm{d} z=-\int_{H}^{z} f_{\mathrm{cd}} \mathrm{d} z$ is the accumulated drag force due to vegetation from $z$ to $H$. According to Eq. (2), when $z \geq h, F_{\mathrm{cd}}=0$; and

$\left(F_{\mathrm{cd}}\right)^{\prime}=\frac{d}{d z}\left(-\int_{H}^{z} f_{\mathrm{cd}} d z\right)=-f_{\mathrm{cd}}$ 
Combining Eqs. (4), (8), (10), (12) and (13) gives

$$
\begin{aligned}
-u \frac{d \tau}{d z} & +u f_{\mathrm{cd}}=\left\{\left[\gamma(H-z) S+\int_{H}^{z} f_{\mathrm{cd}} d z\right] \frac{d u}{d z}+u f_{\mathrm{cd}}\right\} \\
& -\frac{d}{d z}\left(\gamma(H-z) S u+u \int_{H}^{z} f_{\mathrm{cd}} d z\right) .
\end{aligned}
$$

Integrating Eq. (15) from $z$ to $H$ gives

$$
\begin{aligned}
\int_{z}^{H} u( & \left.-\frac{d \tau}{d z}+f_{\mathrm{cd}}\right) d z=\left[\gamma S \int_{z}^{H}(H-z) \frac{d u}{d z} d z+\int_{z}^{H} \int_{H f \mathrm{~cd}}^{z} d z \frac{d u}{d z} d z+\int_{z}^{H} u f_{\mathrm{cd}} d z\right] \\
& -\left\{\int_{z}^{H} d\left[\gamma(H-z) S u+u \int_{H}^{z} f_{\mathrm{cd}} d z\right]\right\} .
\end{aligned}
$$

The left side of Eq. (16) is the energy borrowed from the local flow from $z$ to $H$. Using the control Eq. (1), the left side is rewritten as $\gamma S \int_{z}^{H} u \mathrm{~d} z$. On the right side of Eq. (16), the first and second terms are the total energy spending due to water viscosity from $z$ to $H$, the third term is the energy loss due to vegetation from $z$ to $H$, and the fourth and fifth terms are the total energy transition from $z$ to $H$. Using the definition of $F_{\mathrm{cd}}$, the second term is written as

$\int_{z}^{H} \int_{H}^{z} f_{\mathrm{cd}} d z \frac{d u}{d z} d z=\int_{z}^{H}\left(\int_{H}^{z} f_{\mathrm{cd}} d z\right) d u=-\int_{z}^{H} F_{\mathrm{cd}} d u$

and the fourth and fifth terms are written as

$$
\begin{aligned}
& \int_{z}^{H} d\left[\gamma(H-z) S u+u \int_{H}^{z} f_{\mathrm{cd}} d z\right]=-\gamma S u(H-z)-u\left(\int_{H}^{z} f_{\mathrm{cd}} d z\right) \\
& \quad=-\gamma S u(H-z)+u F_{\mathrm{cd}} .
\end{aligned}
$$

Therefore, Eq. (16) gives

$$
\gamma S \int_{z}^{H} u d z=\left[\gamma S \int_{z}^{H}(H-z) d u-\int_{z}^{H} F_{\mathrm{cd}} d u+\int_{z}^{H} u f_{\mathrm{cd}} d z\right]+\left[\gamma S u(H-z)-u F_{\mathrm{cd}}\right]
$$

or

$$
\left.\left.\left.\left.\left.\left.W_{\mathrm{b}}\right]_{z}^{H}=\left(W_{\mathrm{sw}}\right]_{z}^{H}+W_{\mathrm{sv}}\right]_{z}^{H}\right)+W_{\mathrm{t}}\right]_{z}^{H}=W_{\mathrm{s}}\right]_{z}^{H}+W_{\mathrm{t}}\right]_{z}^{H} .
$$

The physical meanings of each term in Eqs. (19) and (20) are as follows.

(1) $\left.\gamma S \int_{z}^{H} u \mathrm{~d} z=W_{\mathrm{b}}\right]_{z}^{H}$ is the aggregate energy borrowing from $z$ to the water surface. This term is the same as that for flow without vegetation.

(2) $\gamma S \int_{z}^{H}(H-z) \mathrm{d} u$ is the aggregate energy spending that ought to be consumed in the flow without vegetation. In the vegetated flow, the vegetation may restrict the flow turbulence and decrease the spending; therefore, the difference $\gamma S \int_{z}^{H}(H-z) \mathrm{d} u-$ $\left.\int_{z}^{H} F_{\mathrm{cd}} \mathrm{d} u=W_{\mathrm{sw}}\right]_{z}^{H}$ is the energy spending that is consumed by water viscosity in vegetated flow.

(3) $\left.\int_{z}^{H} u f_{\mathrm{cd}} \mathrm{d} z=W_{\mathrm{sv}}\right]_{z}^{H}$ is the aggregate energy spending due to the interaction between flow and vegetation, which indicates that the vegetation will make the flow more turbulent. The term $\left.\left.\left.W_{\mathrm{s}}\right]_{z}^{H}=W_{\mathrm{sw}}\right]_{z}^{H}+W_{\mathrm{sv}}\right]_{z}^{H}$ is the total energy spending from $z$ to $H$ in the flow with vegetation.

(4) $\gamma \mathrm{Su}(\mathrm{H}-\mathrm{z})$ is the aggregate energy transition from $z$ to $H$. But the vegetation may enforce energy transmitted upward to the top of the vegetation. Therefore, $\left.\gamma S u(H-z)-u F_{\mathrm{cd}}=W_{\mathrm{t}}\right]_{z}^{H}$ is the total energy transition in the vegetated flow.

As shown above, the energy borrowed local flow between $z$ and $H$ balances with the energy spending and energy transition between $z$ and $H$. To further discuss the balance relationships, all terms in

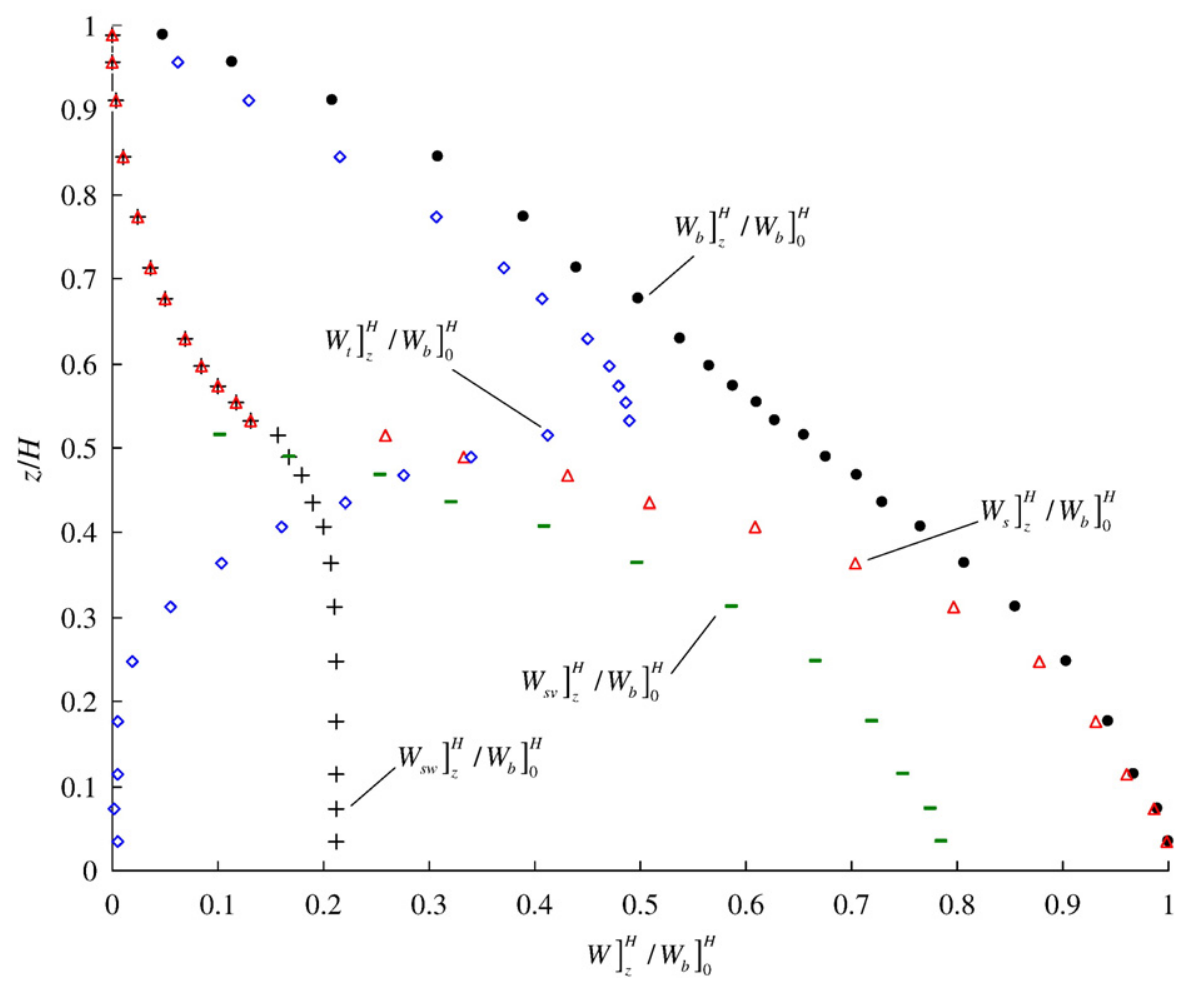

Fig. 3. Accumulative values of the total energy, energy loss and energy transition in a cross-section. 
Eq. (20) are scaled with the total energy borrowing in the whole cross-section $\left.W_{\mathrm{b}}\right]_{0}^{H}$ and plotted as energy accumulative curves in Fig. 3. In the figure, the energy transition accumulative curve reaches its maximum value at the top of the vegetation, and then decreases to zero at the flume bed. Therefore, the energy transition in a whole cross-section is zero, and the energy spending accumulative curve increases slowly in the non-vegetated region but sharply in the vegetated region, and meets the energy borrowing accumulative curve at the bed. Hence, the total energy spending is equal to the total energy borrowing in the whole cross-section. These conclusions are consistent with the results in Section 4.1. The second conclusion is more obvious if the terms in Eq. (19) are calculated from the bed. When $z=0, u=0, \gamma S u(H-z)=0$, and $u F_{\mathrm{cd}}=0$, the total energy transition in the whole cross-section is zero: $\left.W_{\mathrm{t}}\right]_{0}^{H}=0$; therefore, Eq. (20) becomes

$\left.\left.W_{\mathrm{b}}\right]_{0}^{H}=W_{\mathrm{s}}\right]_{0}^{H}$.

Eq. (21) indicates that the total energy borrowing is equal to the total energy spending in the whole-flow region of a uniform flow.

\subsection{Discussion}

Three impacts of vegetation acting on flow have been mentioned: the turbulence restriction due to vegetation $\int_{z}^{H} F_{\mathrm{cd}} \mathrm{d} u$, the turbulence source due to vegetation $\int_{z}^{H} u f_{c d} \mathrm{~d} z$, and the energy transference due to vegetation $u F_{\text {cd }}$. The first two terms are contradictions; the former restricts flow turbulence while the latter increases turbulence. The two terms are usually unbalanced, with the former being smaller than the latter. We perform integration by parts on the first term with Eq. (14):

$$
\begin{aligned}
\int_{z}^{H} F_{\mathrm{cd}} d u & =\left.u F_{\mathrm{cd}}\right|_{z} ^{H}-\int_{z}^{H} u\left(F_{\mathrm{cd}}\right)^{\prime} d z \\
& =-u F_{\mathrm{cd}}+\int_{z}^{H} u f_{\mathrm{cd}} d z
\end{aligned}
$$

or

$\int_{z}^{H} u f_{\mathrm{cd}} d z-\int_{z}^{H} F_{\mathrm{cd}} d u=u F_{\mathrm{cd}}=u \int_{z}^{H} f_{\mathrm{cd}} d z \geq 0$.

Eq. (23) indicates that the turbulence source is usually greater than or equal to the turbulence restriction, which means that overall vegetation increases turbulence. In particular, when $z=0, u=0$, and $u F_{\mathrm{cd}}=0$, the transition term in a whole cross-section only redistributes energy. On the basis of Eq. (23), $\int_{0}^{H} u f_{\mathrm{cd}} \mathrm{d} z=\int_{0}^{H} F_{\mathrm{cd}} \mathrm{d} u$, the turbulence due to vegetation is balanced by the turbulence restriction of vegetation in the whole cross-section.

\section{Conclusions}

This paper gives the expressions of energy borrowed from local flow (Eq. (4)), energy spending (Eq. (8)) and energy transition (Eq. (10)) in an open-channel flow with submerged vegetation. On this basis, the paper discusses the energy balance (Eqs. (12) and (20)) in the vegetated flow and analyzes the effects of vegetation on the flow.

1. Energy spending is concentrated in the vegetated region, especially just below the top of the vegetation. The exchanges of momentum and energy in the upper vegetated region are more intensive than those in the lower vegetated region.
2. Energy borrowed from local flow is greater than the energy spending in the non-vegetated region; on the contrary, the energy borrowing is smaller in the vegetated region. The redundant energy accumulated in the non-vegetated region is transferred to balance the energy spending in the vegetated region.

3. The energy borrowing is equal to the sum of local energy spending and local energy transition at any point of vegetated flow. In the region from $z$ to $H$, the aggregate energy borrowing equals the sum of aggregate energy spending and energy transition. In the wholeflow region, the energy transition accumulation is zero and the total energy borrowing is equal to the total energy loss. These conclusions are similar to those made about flow without vegetation.

4. The effects of vegetation on the flow include the restriction of turbulence by vegetation, turbulence due to vegetation, and transition. The turbulence due to vegetation is the main effect of vegetation acting on the flow.

These conclusions describe how the energy in vegetated flow distributes, transports and dissipates, and are helpful in illustrating how vegetation influences flow. However, we do not strictly distinguish the average height of flexible vegetation and that of rigid vegetation, so the flexibility of vegetation cannot be considered as an influencing factor in the study. This problem warrants further study.

\section{Acknowledgments}

The authors are grateful for the financial support from the Natural Science Foundation of China (Nos. 50679061, 50709025).

\section{References}

[1] Bakhmeteff BA, Allen W. The mechanism of energy loss in fluid friction. Trans, ASCE 1945;111:1043-80.

[2] Cui J, Neary VS. LES study of turbulent flows with submerged vegetation. J Hydraul Res 2008;46(3):307-16.

[3] Ghisalberti M, Nepf HM. Mixing layers and stem-scale coherent structures in vegetated aquatic flows. J Geophys Res 2002;107(C2):3011, doi:10.1029/ 2001JC000871.

[4] Green JC. Modeling flow resistance in vegetated streams: review and development of new theory. Hydrol Process 2005;19(6):1245-59.

[5] Huai WX, Zeng YH, Xu ZG, Yang ZH. Three-layer model for vertical velocity distribution in open channel flow with submerged rigid vegetation. Adv Water Resour 2009;32(4):487-92.

[6] Kouwen N, Fathi-Moghadam M. Friction factors for coniferous trees along rivers. J Hydraul Eng 2000;126(10):732-40.

[7] Kubrak E, Kubrak J, Rowinski PM. Vertical velocity distributions through and above submerged, flexible vegetation. Hydrol Sci J 2008;53(4):905-19.

[8] Nepf HM, Ghisalberti M. Flow and transport in channels with submerged vegetation. Acta Geophysica 2008;56(3):753-77.

[9] Nikora V, Larned S, Nikora N, Debnath K, Cooper G, Reid M. Hydraulic resistance due to aquatic vegetation in small streams: field study. J Hydraul Eng 2008;134 (9):1326-32.

[10] Rowinski PM, Kubrak J. A mixing-length model for predicting vertical velocity distribution in flows through emergent vegetation. J Hydrol Sci 2002;47(6): 893-904.

[11] Shimizu Y, Tsujimoto T. Numerical analysis of turbulent open-channel flow over vegetation layer using a $\kappa-\varepsilon$ turbulence model. J Hydrosci Hydraul Eng 1994;11 (2):57-67.

[12] Thom A. Momentum absorption by vegetation. Q J Roy Meteorol Soc 1971;97 (414):414-28.

[13] Velasco D, Bateman A, Medina V. A new integrated, hydro-mechanical model applied to flexible vegetation in riverbeds. J Hydraul Res 2008;46(5):579-97.

[14] Wilson CAME, Stoesser T, Bates PD, Pinzen AB. Open channel flow through different forms of submerged flexible vegetation. J Hydraul Eng 2003;129(11): 847-53. 